\title{
UNA EXPERIENCIA DE EVALUACIÓN ALTERNATIVA EN UN PROYECTO INTERDISCIPLINAR DE JUEGOS TRADICIONALES EN EDUCACIÓN PRIMARIA
}

An experience of alternative assessment in an interdisciplinary project of traditional games in primary school

Uma experiência de avaliação alternativa num projeto interdisciplinar de jogos tradicionais na educação primária

\section{Isabel Pérez-Herráez (1)}

Alexandra Valencia-Peris (2)

(1) Universitat de València, España. Teléfono:+34 963828920. Correo electrónico: ipehe2@alumni.uv.es (2) Universitat de València, España. Teléfono: +34 963828920. Correo electrónico: alexandra.valencia@uv.es

\section{Resumen}

En este trabajo se describe y analiza una experiencia educativa basada en un proyecto interdisciplinar cuya evaluación se plantea de un modo alternativo, haciendo uso de fichas de seguimiento mediante técnicas de observación (heteroevaluación), autoevaluación y co-evaluación. La propuesta, ha sido realizada gracias a la participación del alumnado de $6^{\circ}$ de primaria, durante las sesiones de Educación Física, trabajando los juegos tradicionales valencianos interdisciplinariamente junto con Lengua y la Literatura, Matemáticas y Educación Artística. La experiencia se ha evaluado con una encuesta y una asamblea final abierta. El alumnado ha mostrado cierta resistencia inicial al involucrarse en procesos participativos de evaluación, aunque un 73,9\% manifiesta que le ha gustado trabajar con esta nueva metodología. Esta propuesta innovadora ha resultado efectiva, sostenible y replicable en otros contextos similares aunque, por el contrario, ha requerido de un alto grado de implicación para la profesora que la ha dinamizado.

Palabras clave: Interdisciplinariedad; participación; evaluación formativa; evaluación compartida; Educación Primaria

\footnotetext{
Abstract

This paper describes and analyses an educational experience based on an interdisciplinary project whose evaluation has been conducted in an alternative way, making use of 
monitoring records through observation techniques (heteroevaluation), self-assessment and co-assessment. 6th grade students took part in the experience along Physical Education lessons, working the traditional Valencian games interdisciplinarily with Language and Literature, Mathematics and Art Education. The experience was evaluated through a survey and an open final assembly. The students have shown some initial resistance when taking part in participatory evaluation processes, although $73.9 \%$ stated that they liked to work with this new methodology. This innovative proposal has been effective, sustainable and replicable in other similar contexts, although, on the contrary, it has been high demanding for the teacher who had developed the experience.

Key words: Interdisciplinarity; participation; formative evaluation; shared evaluation; Primary education

\section{Resumo}

Neste trabalho descreve-se e analisa-se uma experiência educativa baseada num projeto interdisciplinar cuja avaliação se organizade forma alternativa, utilizando fichas de acompanhamento, através de técnicas de observação (heteroavaliação), autoavaliação e coavaliação. A proposta foi realizada graças à participação dos alunos do $6 .^{\circ}$ ano da educação básica, durante as sessões de Educação Física, tendo trabalhando interdisciplinarmente os jogos tradicionais valencianos, em conjunto, com Língua e Literatura, Matemática e Educação Artística. A experiência foi avaliada através de um questionárioe umcírculo final de reflexão aberta. Os alunos mostraram alguma resistência inicial ao participar nos processos de avaliação participativa, embora 73,9\% afirmem ter gostado de trabalhar com esta nova metodologia. Esta proposta inovadora tem-se revelado eficaz, sustentável e replicável a outros contextos semelhantes, embora, por outro lado, tenha exigido um elevado grau de envolvimento por parte da professora que a dinamizou. Palavras-chave: Interdisciplinaridade; participação; avaliação formativa; avaliação partilhada; Educação Primária

\section{Introducción}

La evaluación alternativa hace referencia a todos los sistemas de evaluación que intentan superar a la metodología tradicional de evaluación, basada en la simple realización de pruebas objetivas, con la finalidad única o principal de calificar, como es la evaluación formativa y compartida (López-Pastor \& Pérez-Pueyo, 2017). Por otro lado, los proyectos 
interdisciplinares permiten al alumnado aprovechar las conexiones existentes entre diversas materias y manejar su convergencia cara a un objetivo compartido (Peixoto, 2014). En este trabajo se describe y analiza un ejemplo de modelo de evaluación alternativa llevado a cabo en el desarrollo de un proyecto interdisciplinar sobre juegos tradicionales que trata de fomentar la autonomía y responsabilidad en el alumnado de educación primaria.

\section{Contextualización}

La experiencia educativa se desarrolló en tres grupos de $6^{\circ}$ de primaria de un centro público de educación primaria situado en un pequeño pueblo de la Comunidad Valenciana. El peso del proyecto interdisciplinar recayó principalmente en la asignatura de Educación Física aunque también abordaba contenidos curriculares de las asignaturas de Lengua y Literatura, Matemáticas y Educación Artística.

\section{Diseño y desarrollo}

La propuesta nace a partir de una celebración anual que organiza el centro educativo para dar a conocer los juegos tradicionales valencianos. Hasta ahora se realizaba de manera independiente de la programación curricular. En ella, el alumnado de $6^{\circ}$ de Primaria, quienes ejercían el rol de monitores y monitoras, se encargaban de organizar los juegos tradicionales para el resto de cursos en un día de la semana cultural. Sin embargo, en esta ocasión, en vez de ser los y las docentes de la asignatura los encargados de escoger los juegos que se practican durante la celebración de la jornada es el alumnado quien, desde un proyecto interdisciplinar y a partir de las aportaciones de familiares, amistades y/o cualquier persona conocida, realiza una tarea de búsqueda y recopilación de aquellos juegos que consideren más interesantes para enseñar a los compañeros y compañeras de otros cursos. Durante todo el proceso el alumnado va evaluando su capacidad de transmisión y organización de los juegos mediante técnicas de coevaluación y autoevaluación grupales. El sistema de evaluación empleado se detalla en la Tabla 1.

Con el fin de conocer las percepciones del alumnado y su satisfacción con el proyecto interdisciplinar, se administró un cuestionario con respuestas tipo Likert y se realizó una asamblea final abierta.

Una experiencia de evaluación alternativa en un proyecto interdisciplinar de juegos tradicionales en 
Tabla 1.

Resumen de actividades y técnicas y su relación con los instrumentos de evaluación utilizados en el proyecto interdisciplinar.

\begin{tabular}{|c|c|c|c|}
\hline \multicolumn{3}{|c|}{ EVALUACIÓN } & Calificación \\
\hline $\begin{array}{l}\text { Actividades de } \\
\text { aprendizaje }\end{array}$ & $\begin{array}{l}\text { Técnica de } \\
\text { evaluación }\end{array}$ & Instrumento & $\%$ \\
\hline $\begin{array}{l}\text { Práctica supervisada } \\
\text { en cada sesión }\end{array}$ & $\begin{array}{l}\text { Observación. } \\
\text { Heteroevaluación. }\end{array}$ & $\begin{array}{l}\text { Ficha de seguimiento grupal para } \\
\text { valorar contenidos actitudinales. } \\
\text { Ficha de seguimiento grupal para } \\
\text { valorar contenidos procedimentales y } \\
\text { conceptuales. }\end{array}$ & 40 \\
\hline $\begin{array}{l}\text { Trabajo escrito de } \\
\text { recopilación juegos } \\
\text { tradicionales }\end{array}$ & $\begin{array}{l}\text { Análisis } \\
\text { documental. } \\
\text { Heteroevaluación. }\end{array}$ & $\begin{array}{l}\text { Ficha de seguimiento grupal sobre la } \\
\text { recopilación y explicación de juegos } \\
\text { tradicionales. }\end{array}$ & 10 \\
\hline $\begin{array}{l}\text { Demostración y } \\
\text { explicación del juego } \\
\text { tradicional }\end{array}$ & $\begin{array}{l}\text { Autoevaluación } \\
\text { grupal }\end{array}$ & $\begin{array}{l}\text { Ficha de autoevaluación grupal sobre la } \\
\text { explicación del juego. }\end{array}$ & 10 \\
\hline $\begin{array}{l}\text { Exposición de los } \\
\text { juegos tradicionales }\end{array}$ & $\begin{array}{l}\text { Co-evaluación } \\
\text { grupal }\end{array}$ & $\begin{array}{l}\text { Ficha de coevaluación grupal para } \\
\text { evaluar la exposición de otros grupos. }\end{array}$ & 10 \\
\hline
\end{tabular}

\section{Evaluación}

Tras realizar la experiencia e interpretar los resultados derivados de la investigación realizada, se extraen las siguientes generalizaciones:

- Aunque podemos encontrar alguna excepción, habitualmente el alumnado se autoevalúa con sinceridad. Esto puede deberse al hecho de que el alumnado ha logrado asociar la evaluación con una función formativa, como una oportunidad de mejora y no como una sentencia que no tiene vuelta atrás.

- Es de agradecer la participación del alumnado en este tipo de procesos ya que, en ocasiones incluso nos aportan informaciones que se pueden haber pasado por alto al profesorado. Así, se trata de promover en el alumnado la asunción de responsabilidades implicándoles en su propia evaluación, garantizando así una mayor regulación de su trabajo (autonomía y responsabilidad) e implicación en el día a día (Hortigüela, PérezPueyo \& Fernández-Río, 2017).

- El alumnado ha mostrado cierta resistencia inicial al involucrarse en procesos participativos de evaluación, aunque un $73,9 \%$ manifiesta que le ha gustado trabajar con esta nueva metodología los juegos populares.

- La falta de tiempo y el ritmo acelerado dificulta el aprovechamiento de los beneficios de la evaluación formativa. Del análisis de las respuestas se desprenden algunas 
contradicciones que llevan a pensar que no se ha comprendido cuál era la pregunta y tampoco ha habido oportunidad de resolver estas dudas.

\section{Conclusiones}

En el proyecto interdisciplinar llevado a cabo para trabajar los juegos tradicionales se ha empleado un modelo de evaluación poco convencional en el contexto en el que se ha desarrollado, en el que se ha utilizado la evaluación formativa y compartida. Así, se ha pretendido involucrar al máximo al alumnado tanto en el diseño y ejecución de las actividades como en la evaluación de las mismas y en su propio proceso de aprendizaje. Esto, que inicialmente puede parecer beneficioso desde un punto de vista teórico, a la hora de llevarlo a la práctica con alumnado poco acostumbrado a participar en su evaluación puede acarrear algún inconveniente, el cual se va subsanando conforme el estudiantado se va habituando a esta nueva metodología.

En definitiva, esta propuesta innovadora parece resultar efectiva, sostenible y replicable en otros contextos similares si bien hay que tener en cuenta el alto grado de implicación que exige por parte del profesorado que decida ponerla en marcha. Asimismo, se prevé sustituir las fichas de observación, autoevaluación y co-evaluación por rúbricas, donde los niveles de logro queden mejor definidos y sean más útiles tanto para el alumnado como para el profesorado.

\section{Referencias}

Hortigüela, D., Pérez-Pueyo, Á., \& Fernández-Río, J. (2017). Relación entre el estilo actitudinal y la responsabilidad evaluativa del alumnado de Educación Física. Cultura, Ciencia y Deporte, 12, 89-99.

López-Pastor, V., \& Pérez-Pueyo, Á. (Coords.) (2017). Buenas prácticas docentes. Evaluación formativa y compartida en educación: experiencias de éxito en todas las etapas educativas. León: Universidad de León.

Peixoto, L. (2014). Proyecto interdisciplinar, «escuela: comunidad olímpica». Retos: nuevas tendencias en educación física, deporte y recreación, 25, 140-143. 